\title{
Über ein quantenmechanisches Näherungsverfahren zur Behandlung der $\pi$-Elektronensysteme aromatischer Kohlenwasserstoffe II
}

\author{
Von Hermann Hartmann \\ Aus dem Institut für physikalische Chemie der Universität Frankfurt a. M. \\ (Z. Naturforschg. 2 a, 263-264 [1947]; eingegangen am 17. Januar 1947)
}

Die Resultate des vorstehend ${ }^{1}$ veröffentlichten Näherungsverfahrens zur quantenmechanischen Berechnung der Energie des $\pi$-Elektronensystems aromatischer Kohlenwasserstoffe werden mit denen des $\mathrm{H} \ddot{u} \mathrm{ck}$ el schen Näherungsverfahrens verglichen. Bei den 18 Molekülen, die zum Vergleich herangezogen werden konnten, ergab sich sehr gute Utbereinstimmung.

$\mathrm{D}$ ie Resultate des vorstehend ${ }^{\mathbf{1}}$ veröffentlichten Näherungsverfahrens wurden mit den entsprechenden Resultaten des Slater-Paulingschen Näherungsverfahrens verglichen. Wegen der Kompliziertheit der Slater-Pauling-Rechnungen über aromatische Kohlenwasserstoffe standen für diesen Vergleich nur wenige Daten zur Verfügung. Wir haben deshalb weiterhin eínen Vergleich mit den Resultaten des Hü ck el schen Näherungsverfahrens durchgeführt, mit dessen Hilfe wesentlich mehr Moleküle behandelt worden sind ${ }^{2}$.

Unser Näherungsverfahren lieferte für die Energie $E$ des $\pi$-Elektronensystems von Acenen den Ausdruck

$$
E=\left(C+\frac{17}{12} A\right)+n\left(5 C+\frac{19}{12} A\right),
$$

wo $C$ bzw. $A$ das Coulomb-bzw. AustauschIntegral bedeutet und $n$ die Zahl der linear anellierten Sechsringe ist. $E$ ist also eine lineare Funktion von $n$. Nach $\mathrm{H}$ ü ckel gilt für die Energie des $\pi$-Elektronensystems der Acene

$$
E_{H \ddot{u}}=(2+4 n) a+k(n) \beta .
$$

$\alpha$ und $\beta$ sind das $\mathrm{H} \ddot{\mathrm{u}} \mathrm{ckel}$ sche $\mathrm{Cou} \mathrm{lom} \mathrm{b}-$ und das Resonanz-Integral, und $k(n)$ ist eine von $n$ abhängige Größe, über deren Werte Tab. 1' Auskunft gibt.

In die Tab. 1 sind außerdem die Differenzen der aufeinanderfolgenden $k$-Werte eingetragen. Diese Differenzen sind nahezu konstant, so daß auch $E_{H \ddot{u}}$ nahezu eine lineare Funktion von $\boldsymbol{n}$ ist, was nach (1) zu fordern wäre, wenn die

1 H. Hartmann, Z. Naturforschg. 2a, 259 [1947].

2 Wegen der hier benutzten Ergebnisse des $\mathrm{H}$ ü ck el schen Näherungsverfahrens und wegen dieses Verfahrens selbst vergleiche man E. Hü ckel, Grundzüge der Theorie ungesättigter und aromatischer Verbindungen, Berlin 1938.

\begin{tabular}{|l|r|r|c|}
\hline \multicolumn{1}{|c|}{ Stoff } & $n$ & $k(n)$ & \multicolumn{1}{c|}{$\Delta k$} \\
\hline Benzol & $\mathbf{1}$ & $\mathbf{8 , 0 0}$ & \\
Naphthalin & $\mathbf{2}$ & $\mathbf{1 3 , 6 8}$ & $\mathbf{5 , 6 8}$ \\
Anthracen & 3 & 19,31 & 5,63 \\
Tetracen & $\mathbf{4}$ & $\mathbf{2 4 , 9 3}$ & $\mathbf{5 , 6 2}$ \\
Pentacen & $\mathbf{5}$ & $\mathbf{3 0 , 5 2}$ & $\mathbf{5 , 5 9}$ \\
Hexacen & $\mathbf{6}$ & $\mathbf{3 6 , 1 2}$ & $\mathbf{5 , 6 0}$ \\
Heptacen & $\mathbf{7}$ & $\mathbf{4 1 , 7 4}$ & $\mathbf{5 , 6 2}$ \\
& & & \\
\hline
\end{tabular}

Tab. 1. Die H ü c k e l schen Koeffizienten des Resonanzintegrals für die Acene.

Resultate unseres Näherungsverfahrens mit denen des $\mathrm{Hü} \mathrm{ckel} \mathrm{schen} \mathrm{übereinstimmen} \mathrm{sollen.} \mathrm{Gl.} \mathrm{(2)}$ kann in sehr guter Näherung durch die lineare Funktion

$$
E_{H \ddot{u}}^{\prime}=(2 \alpha+2,47 \beta)+n(4 a+5,61 \beta)
$$

ersetzt werden. Setzt man nun $E=E_{H \ddot{u}}$, so folgen zwei Beziehungen zwischen den vier Größen $A$, $C, \propto, \beta$. Man kann mit Hilfe dieser Beziehungen $C$ und $A$ durch $\alpha$ und $\beta$ ausdrücken und erhält

$$
\left.\begin{array}{l}
C=0,3464 \alpha+0,7328 \beta \\
A=1,0928 \alpha+1,2277_{\beta}
\end{array}\right\} .
$$

Ein allgemeiner Vergleich unseres Näherungsverfahrens mit dem $\mathrm{H} \ddot{\mathrm{u}} \mathrm{ckelschen}$ ist jetzt in folgender Weise möglich: Man setzt in unsere allgemeine Beziehung

$$
E=N C+\sum_{i} \frac{N_{i}}{i} A
$$

die Ausdrücke (3) ein und faßt die Glieder mit $\alpha$ und $\beta$ zusammen. So ergibt sich:

$$
\begin{aligned}
E & =\left(0,3464 N+1,0928 \sum_{i} \frac{N_{i}}{i}\right) \\
& +\left(0,7328 N+1,2277 \sum_{i} \frac{N_{i}}{i}\right)_{\beta} .
\end{aligned}
$$


Da für die Gesamtenergie nach $\mathrm{H} \ddot{\mathrm{c}} \mathrm{ckel}$ allgemein gilt

$$
E_{H \ddot{u}}=Z a+l i \beta,
$$

wo $Z$ die Anzahl der $\pi$-Elektronen bedeutet, müßte, wenn unser Näherungsverfahren dieselben

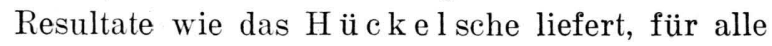
in Frage kommenden Moleküle

$$
k^{\prime}=\left(0,7328 N+1,2277 \sum_{i} \frac{N_{i}}{i}\right)=k
$$

sein. In Tab. 2 haben wir für eine Reihe von Molekülen die $\mathrm{H}$ ückelschen $k$-Werte und die mit unserem Verfahren auf dem angegebenen Weg berechneten $k^{\prime}$-Werte zusammengestellt.

Die Betrachtung der Tab. 2 lehrt, daß unser Näherungsverfahren auch mit dem Hückelschen in sehr guter Übereinstimmung ist.

Tab. 2. Vergleich der $k$ - und $k^{\prime}$-Werte für verschiedene

\begin{tabular}{|c|c|c|c|}
\hline Stoff & Formel & $k$ & $k^{\prime}$ \\
\hline 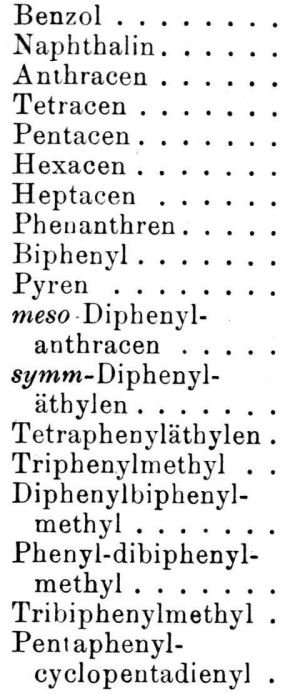 & $\begin{array}{l}\mathrm{C}_{6} \mathrm{H}_{33} \\
\mathrm{C}_{10} \mathrm{H}_{8} \\
\mathrm{C}_{14} \mathrm{H}_{10} \\
\mathrm{C}_{18} \mathrm{H}_{12} \\
\mathrm{C}_{22} \mathrm{H}_{14} \\
\mathrm{C}_{26} \mathrm{H}_{18} \\
\mathrm{C}_{30} \mathrm{H}_{18} \\
\mathrm{C}_{14} \mathrm{H}_{10} \\
\mathrm{C}_{12} \mathrm{H}_{10} \\
\mathrm{C}_{16} \mathrm{H}_{10} \\
\mathrm{C}_{14} \mathrm{H}_{8}\left(\mathrm{C}_{6} \mathrm{H}_{5}\right)_{2} \\
\mathrm{C}_{2} \mathrm{H}_{2}\left(\mathrm{C}_{6} \mathrm{H}_{5}\right)_{2} \\
\mathrm{C}_{2}\left(\mathrm{C}_{6} \mathrm{H}_{5}\right)_{4} \\
\mathrm{C}_{(}\left(\mathrm{C}_{6} \mathrm{H}_{5}\right)_{3} \\
\mathrm{C}\left(\mathrm{C}_{6} \mathrm{H}_{5}\right)_{2}\left(\mathrm{C}_{12} \mathrm{H}_{9}\right) \\
\mathrm{C}\left(\mathrm{C}_{6} \mathrm{H}_{5}\right)\left(\mathrm{C}_{12} \mathrm{H}_{9}\right)_{2} \\
\mathrm{C}_{(}\left(\mathrm{C}_{12} \mathrm{H}_{9}\right)_{3} \\
\left(\mathrm{C}_{6} \mathrm{H}_{5}\right)_{5} \mathrm{C}_{5}\end{array}$ & $\begin{array}{r}8,00 \\
13,68 \\
19,31 \\
24,93 \\
30,52 \\
36,12 \\
41,74 \\
19,45 \\
16,38 \\
22,50 \\
36,17 \\
18,88 \\
35,73 \\
25,79 \\
34,20 \\
\\
42,61 \\
51,01 \\
\\
48,15\end{array}$ & $\begin{array}{r}8,08 \\
13,69 \\
19,29 \\
24,92 \\
30,53 \\
36,13 \\
41,75 \\
19,41 \\
16,38 \\
22,44 \\
36,30 \\
18,97 \\
35,78 \\
26,10 \\
34,43 \\
42,72 \\
51,05 \\
48,77\end{array}$ \\
\hline
\end{tabular}
Moleküle.

\title{
Resonanz und Wasserstoffbindung der Carboxyl- und der Amidgruppe
}

\author{
Von KarL WirTz \\ Aus dem Max-Planck-Institut für Physik, Göttingen \\ (Z. Naturforschg. 2 a, 264-271 [1947]; eingegangen am 17. Januar 1947)
}

Moleküle mit Carboxyl- und Amidgruppen gehen untereinander besonders feste Wasserstoffbindungen ein. Das bekannteste Beispiel sind die Doppelmoleküie der Carbonsäuren. Wie bei allen Wasserstoffbindungen besteht der Hauptteil der Bindungsenergie aus elektrostatischer Anziehung der gegenüberliegenden Dipole. Außerdem muß man jedoch einen bisher unbekannten, nicht elektrostatischen Anteil annehmen, um die beobachteten Bindungsenergien zu verstehen. Er hängt mit den besonderen Bindungsverhältnissen innerhalb der Carboxyl- und Amidgruppe zusammen. Dort besteht echte Resonanz der $\pi$-Elektronen der C-, O- und N-Atome. Diese Resonanz wurde nach dem Verfahren von Hund, Mulliken, Hü ckel untersucht, das auf den Fall erweitert wurde, daß dis $\pi$-Eigenfunktionen verschiedenen Atomen angehören. Die mit Hilfe der Theorie erhaltene Resonanzverfestigung des Carboxylions von rund $40 \mathrm{Kcal}$ stimmt befriedigend mit Werten überein, die aus experimentellen Beobachtungen abgeleitet werden.

Die Resonanz ist in der undissoziierten monomeren Gruppe wegen der Ungleichheit der beiden kanonischen Valenzschemata am unvollkommensten. Sie ist besser, wenn das Molekül eine Wasserstoffbindung eingeht, da dann die Struktur „symmetrisiert“ wird. Am vollkommensten ist sie im Carboxylion, wodurch zur Hauptsache der saure Charakter erklärt wird.

Die Festigkeit der Wasserstoffbindung derartiger Gruppen rührt also zum Teil davon her, daß ihre ,innere“ Resonanz beim Eingehen der Wasserstoffbindung verbessert wird. Es wird versucht, diesen Anteil für das Essigsäure-Doppelmolekül zu bestimmen. Es ergibt sich ein vorläufiger Wert von etwa 2 Kcal pro H-Bindung.

1. Resonanzverfestigung der Carboxyl- und der Amidgruppe

$\mathrm{D}^{\mathrm{c}}$ ie Bindungsenergie normaler Moleküle kann in der Regel aus Energieanteilen der Einzelbindungen additiv zusammengesetzt werden ${ }^{1}$. Die absoluten Werte der einzelnen Bindungsenergien sind bis heute unsicher, da die überall eingehende Sublimationswärme des Diamants unsicher ist ${ }^{2}$.

${ }^{1}$ Vergl. z. B. L. Pauling, The Nature of the Chemical Bond, 2. Aufl. [1940]. 